\title{
Enhancement of FITC Fluorescence by Silver Colloids and Silver Island Films
}

\author{
S. Kruszewski*, T. Wybranowski, M. Cyrankiewicz, \\ B. Ziomkowska AND A. PAWLACZYK
}

Medical Physics Division, Biophysics Department

Collegium Medicum of Nicolaus Copernicus University

Jagiellońska 13, 85-067 Bydgoszcz, Poland

(Received March 27, 2007; in final form February 15, 2008)

\begin{abstract}
Silver colloids and silver island films as fluorescence enhancement systems are the subject of this paper. Enhancement of fluorescence of molecules placed near metallic nanoparticles is a result of resonance interaction of exciting and emitted lights with surface plasmons. Two models explaining this phenomenon are presented. Processes of silver colloids and silver island films forming are described. Results of extinction, absorption and scattering studies of fluorescence enhancement systems are presented. The influence of these systems, i.e. silver colloids and silver island films on the fluorescence of fluorescein-5-isothiocyanate was studied. 7-fold increase in fluorescence intensity of fluorescein-5-isothiocyanate molecules placed near silver nanoparticles was observed.
\end{abstract}

PACS numbers: 33.20.Fb, 33.20.Kf, 33.50.-j

\section{Introduction}

Nanostructures of coinage metals onto which the molecules are adsorbed are able to enhance $10^{6}-10^{12}$-fold the intensity of the Raman scattered light. This phenomenon observed for the first time in 1974 [1] and called surface enhanced Raman scattering (SERS) has become useful tools for chemical, biochemical and biomedical studies [2,3]. Fluorescence and absorption properties of dyes located near SERS-active metallic nanoparticles have been widely studied over the last few years [4-6]. The observed phenomena of fluorescence enhancement called as metal enhanced fluorescence (MEF) seem to be also very promising for biomedical studies [7].

Fluorescein-5-isothiocyanate (FITC) is used in microscopy studies of biological samples and as fluorescent probes in immunoassays [8]. Free FITC exhibits

*corresponding author; e-mail: skrusz@cm.umk.pl 
high fluorescence quantum yield, but fluorescence of FITC bound to proteins is self-quenched, which is undesirable. Self-quenching of FITC fluorescence is due to resonance energy transfer (RET) between FITC molecules (homoRET). Förster distance for homoRET for FITC is about $4.2 \mathrm{~nm}$, this means that if distance between two FITC molecules bound to protein is smaller than $4.2 \mathrm{~nm}$, efficiency of RET is higher than $50 \%[8,9]$. Sizes of many proteins are larger than this distance, therefore the increase in labelled proteins results in the greater efficiency of RET. Colloidal silver was deposited onto glass slides and then was coated with FITC labelled protein. Human serum albumin (HSA) forms monolayer on silvered surface [8]. Fluorescence properties of system FITC-HSA layer on silver island film were studied.

Fluorescence enhancement by metallic nanoparticles is a new phenomenon, studied over last few years $[5-8,10]$. Unique properties of metallic nanoparticles are related with existence of surface plasmons. Plasmons excited in metallic nanoparticles have the characteristic frequencies dependent on sizes, shapes, and dielectric functions of these nanoparticles and surrounding medium [11]. If metallic nanoparticles are in the field of light and if frequency of this light is equal or close to frequency of surface plasmons, in vicinity of these nanoparticles resonance enhancement of electric field of light occurs [12]. If molecules, which are able to absorb the light are placed in this enhanced field, the excitation rate of the molecules increases (from value $\Gamma_{\text {exc }}$ for molecules situated in absence of nanoparticles to larger value $\Gamma_{\mathrm{exc}}^{\mathrm{M}}$ for molecules placed in neighborhood of metallic nanoparticles) [13]. Then, much more molecules will be excited when they are placed near nanoparticles, than whilst they are far from metallic nanoparticles. The enhancement factor of excitation can be determined as

$$
G_{\mathrm{exc}}=\Gamma_{\mathrm{exc}}^{\mathrm{M}} / \Gamma_{\mathrm{exc}} \text {. }
$$

$G_{\text {exc }}$, because of resonance character of this phenomenon, depends on frequency of exciting light and will be maximal if frequency of exciting light is equal to frequency of surface plasmons [13]. Excited molecules can be at least in two way deactivated: nonradiatively with deactivation rate $\Gamma_{\text {nrad }}$ and radiatively by fluorescence light emission with rate $\Gamma_{\text {rad }}$ [12]. Quantum yield of fluorescence (radiative process) is defined as

$$
Q=\frac{\Gamma_{\mathrm{rad}}}{\Gamma_{\mathrm{rad}}+\Gamma_{\mathrm{nrad}}} .
$$

When excited molecules are near metallic nanoparticles, the light emitted by these molecules interacts resonantly with surface plasmons. Intensity of electric field increases, that accelerates process of radiative transitions, radiative deactivation rate $\Gamma_{\text {rad }}^{\mathrm{M}}$ rises, finally quantum yield of fluorescence increases and it is determined as $[12,13]$ :

$$
Q^{\mathrm{M}}=\frac{\Gamma_{\mathrm{rad}}^{\mathrm{M}}}{\Gamma_{\mathrm{rad}}^{\mathrm{M}}+\Gamma_{\mathrm{nrad}}^{\mathrm{M}}} .
$$


The ratio $Q^{\mathrm{M}} / Q=G_{\mathrm{em}}$ can be called as enhancement factor of fluorescence emission. The total enhancement of intensity of fluorescence light near metallic nanoparticles is a result of two resonance enhancements: enhancement of excitation $G_{\mathrm{exc}}$ and enhancement of emission $G_{\mathrm{em}}$ and is equal to $G=G_{\mathrm{exc}} G_{\mathrm{em}}$. It is largest if excitation and emission lights are in (or close to) resonance with surface plasmons excited in nanoparticles. Process of fluorescence enhancement, in contrast to the Raman scattering enhancement, occurs when fluorescence molecules are placed close but usually far than $5 \mathrm{~nm}$ from metallic nanoparticles [7, 14]. Fluorescence of molecules which are adsorbed at nanoparticles or are situated closer than $5 \mathrm{~nm}$ from nanoparticles is quenched. It is caused by nonradiative energy transfer from excited molecule to metal $[15,16]$.

Authors of papers [17-20] proposed another model explaining the mechanism of fluorescence enhancement. This model assumes that the excited molecules placed near metallic nanoparticle transfer energy to metal and excite the surface plasmons. Excited plasmons emit light with frequency of molecule fluorescence [19]. It is resonant process, then intensity of emitted light is larger than fluorescence of molecules situated in medium free of metallic nanoparticles. Authors $[17,19]$ of this "radiating plasmons" model claim that for occurring large fluorescence enhancement, the strong scattering of light on nanoparticles is necessary. They state that the largest enhancement occurs, when the scattering spectrum of enhancement system exhibits maximum at frequency of maximal fluorescence and when the scattering exceeds the absorption (cross-section for scattering on metallic nanoparticles should be larger than cross-section for absorption).
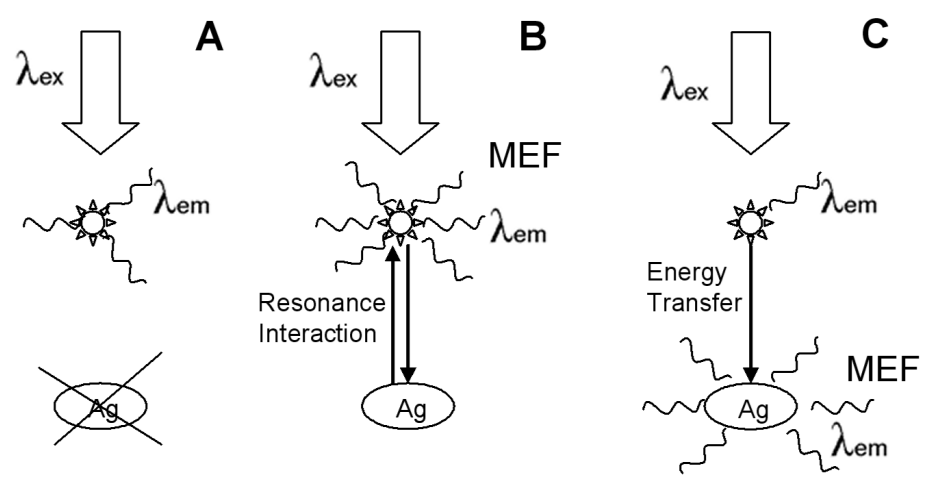

Fig. 1. Scheme of fluorescence of free molecule (A), metal enhanced fluorescence according to model of resonance interaction of excited and emitted lights with surface plasmons (B), metal enhanced fluorescence according to model of "radiating plasmons" (C).

Figure 1 shows schematically the phenomenon of fluorescence of free molecules i.e. in absence of the metallic nanoparticles (Fig. 1A) as well as the phenomenon of fluorescence enhancement by metallic nanoparticles (Figs. 1B 
and $\mathrm{C}$ ). Figure $1 \mathrm{~B}$ interprets the MEF phenomenon as a result of resonance interaction of exciting and emitted lights with surface plasmons, which leads to the increase in intensity of fluorescence light emitted by molecules being in vicinity of metallic nanoparticles. Figure 1C interprets MEF phenomenon as a result of "radiating plasmons" mechanism - light is emitted mainly by surface plasmons - it has frequency of fluorescence light and its intensity is resonantly enhanced.

\section{Materials and methods}

Silver nitrate, trisodium citrate, ethanol and ammonia solution (25\%) were obtained from POCH S.A. FITC was purchased from Sigma-Aldrich. 3-aminopropyltriethoxysilane (APS) was obtained from Fluka. The silver colloids used in these experiments were prepared by chemical reduction of silver nitrate with trisodium citrate using a modified Lee and Meisel method [21-23]. According to this method the sodium citrate first acts as a reducing agent, and later the negative citrate ions are adsorbed on silver nanoparticles. The layer of citrate anions prevents aggregation of nanoparticles. This procedure leads to obtaining nanoparticles, which are approximately spherical and uniform in size and stable during several weeks $[24,25]$.

For obtaining the colloid with relatively small nanoparticles the following procedure, called as procedure I, has been used. $0.1 \mathrm{~g}$ trisodium citrate was dissolved in $10 \mathrm{ml}$ of deionized water. Then $1 \mathrm{ml}$ of this solution was added to $50 \mathrm{ml}$ of heated $\left(90^{\circ} \mathrm{C}\right) 1 \mathrm{mM}$ aqueous solution of $\mathrm{AgNO}_{3}$ and the mixture was warmed up to $95^{\circ} \mathrm{C}$ and stirred for $60 \mathrm{~min}$. The suspension of silver particles was formed. It was next purified by centrifugation at $5000 \mathrm{rpm}$ for $8 \mathrm{~min}$ and the precipitate was dissolved in $10 \mathrm{ml}$ of $1 \mathrm{mM}$ trisodium citrate. The colloid was obtained and it was named as colloid I.

For obtaining the colloid with relatively larger nanoparticles the procedure II has been used. $0.1 \mathrm{~g}$ trisodium citrate was dissolved in $10 \mathrm{ml}$ of deionized water. Then $0.1 \mathrm{ml}$ of this solution was added to $50 \mathrm{ml}$ of heated $\left(60^{\circ} \mathrm{C}\right) 1 \mathrm{mM}$ aqueous solution of $\mathrm{AgNO}_{3}$. The mixture was warmed up to $95^{\circ} \mathrm{C}$ and stirred for $20 \mathrm{~min}$. Then $0.9 \mathrm{ml}$ of aqueous solution of trisodium citrate was added again to the mixture in 3 portions every $15 \mathrm{~min}$. The obtained suspension was purified by centrifugation at $5000 \mathrm{rpm}$ for $8 \mathrm{~min}$ and the precipitate was dissolved in $10 \mathrm{ml}$ of $1 \mathrm{mM}$ trisodium citrate, and in this way the colloid was formed. It was called as colloid II.

The colloidal silver nanoparticles were deposited onto the glass slides by immersing the slides in the colloid for $16 \mathrm{~h}$. In this way the silver island film was formed on the glass. Prior to coating, the glass slides were dipped for $30 \mathrm{~min}$ in $1 \%$ aqueous solution of APS.

The $10 \mu \mathrm{M}$ aqueous solution of FITC-HSA (pH 7.4) with molar ratio of 9:1 was prepared. The glass slides and glass slides covered by silver nanoparticles were soaked for $40 \mathrm{~h}$ in prepared solution of FITC-HSA and in this way the monolayers of FITC-HSA were formed on the slides. 


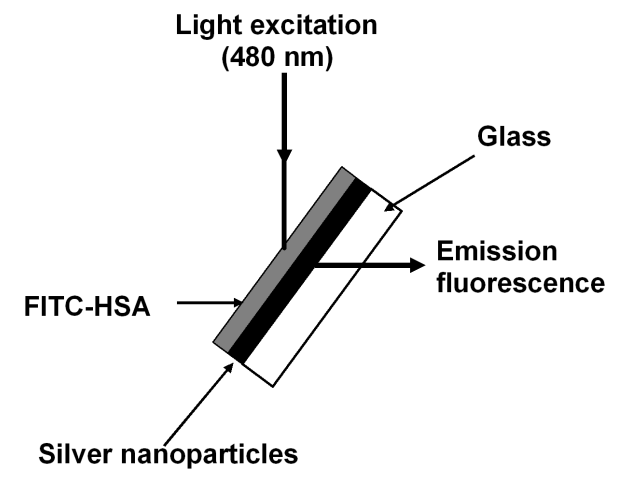

Fig. 2. Experimental sample geometry for metal enhanced fluorescence.

The spectrophotometer SPECORD UV/VIS (Carl Zeiss) was used for recording extinction spectra of the colloids and silver island films. The modified QM-3 steady state spectrofluorometer (Photon Technology International - PTI) was used for recording fluorescence emission spectra of FITC in the colloid, FITCHSA layer on glass and FITC-HSA on silver island film. The light of $480 \mathrm{~nm}$ wavelength was used for fluorescence excitation. Figure 2 presents the sample geometry used for recording fluorescence spectra of FITC-HSA layers. PTI spectrofluorometer equipped with linear polarizer and analyzer - both adjusted to polarize light perpendicularly to the plane of observation - was also used for intensity measurements of light scattered on the colloids. Colloid placed in the sample compartment of spectrofluorometer was illuminated by light from excitation monochromator and intensity of perpendicularly scattered light in function of wavelength was measured, i.e. scattering spectrum of colloid recorded.

\section{Results and discussion}

On the basis of extinction spectra of colloids recorded by spectrophotometer and scattering spectra of these colloids recorded by spectrofluorometer with measurement geometry described in previous section the spectra of cross-sections for extinction and scattering were obtained and next spectrum of cross-section for absorption was calculated. These 3 spectra of optical cross-sections for two colloids studied in this work are presented in Fig. 3A and B. These spectra were determined upon the following premises (see [26]): (1) extinction $E(\lambda)$ informs on decrease in light intensity caused by absorption and scattering, (2) intensity of scattered light $I_{\mathrm{S}}(\lambda)$ is proportional to the cross-section for scattering $C_{\mathrm{S}}(\lambda)$ and extinction $E(\lambda)$ is proportional to the cross-section for extinction $C_{\mathrm{E}}(\lambda),(3)$ the red light is not absorbed by colloid, it is only scattered, then one can assume that $C_{\mathrm{E}}(700 \mathrm{~nm})=C_{\mathrm{S}}(700 \mathrm{~nm}),(4)$ cross-section for extinction is equal to the sum of cross-sections for absorption and scattering $C_{\mathrm{E}}(\lambda)=C_{\mathrm{A}}(\lambda)+C_{\mathrm{S}}(\lambda)$. Figure $3 \mathrm{~A}$ presents above mentioned spectra of colloid I and Fig. 3B the analogical spectra of 
colloid II. The significant differences exist in extinction, absorption and scattering spectra of colloid I and colloid II. Coloid I exhibits the large scattering as well as large absorption in spectral region $400-500 \mathrm{~nm}$, and cross-section for scattering is larger than for absorption, but difference is not large - scattering does not contribute dominantly to extinction. Colloid I exhibits maximum of absorption at $425 \mathrm{~nm}$, maximum of scattering at $440 \mathrm{~nm}$ and maximum of extinction at about $435 \mathrm{~nm}$, which permits to conclude (basing on the results presented in [26]) that average radius of nanoparticles forming colloid I is equal to $35 \mathrm{~nm}$.

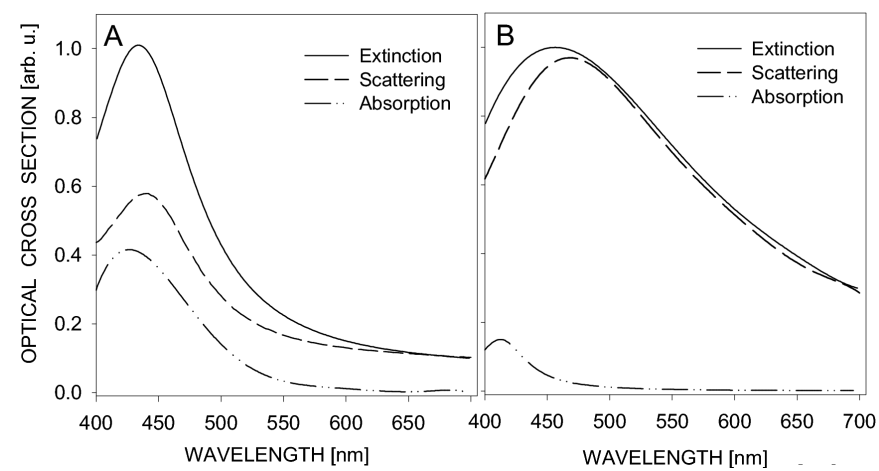

Fig. 3. Extinction, absorption and scattering spectra of 5-fold water diluted colloid I (radius of nanoparticles $35 \mathrm{~nm}$ ) (A) and colloid II (radius of nanoparticles $45 \mathrm{~nm}$ ) (B).

For the colloid II, as is shown in Fig. 3A, the dominant component of extinction in whole spectral region is scattering. Measurable absorption is observed only in spectral region $400-470 \mathrm{~nm}$, and its contribution to extinction is significantly lower than scattering. This colloid exhibits maxima of absorption, scattering and extinction at 410, 470, and $455 \mathrm{~nm}$, respectively, which points out (according to [26]) that average radius of silver nanoparticles in this colloid is equal to 45 $\mathrm{nm}$. The essential difference in scattering spectra of colloid I and colloid II is their width. Scattering spectrum of colloid II (average radius of nanoparticles $45 \mathrm{~nm}$ ), as is shown in Fig. 3B, is very wide.

Figure 4 shows extinction spectra of silver islands deposited on glass by $16 \mathrm{~h}$ dipping of glass slides in colloid I (Fig. 4A) and in colloid II (Fig. 4B). The similarity of spectrum from Fig. 4A to suitable spectrum from Fig. 3A permits to suppose that the extinction of island film obtained from colloid I is contributed in comparable amounts by absorption and scattering, like as it is for colloid I. Maximum of extinction of this island film, like as for colloid I, occurs at about 430 $\mathrm{nm}$, which permits to conclude that during deposition of nanoparticles on glass slide the aggregation does not occur, or occurs in very small degree.

The similarity of extinction spectrum (Fig. 4B) of island film obtained from colloid II to extinction spectrum of colloid II (Fig. 3B) permits to conclude that like as for colloid II, the scattering is the dominant component of extinction. Ex- 


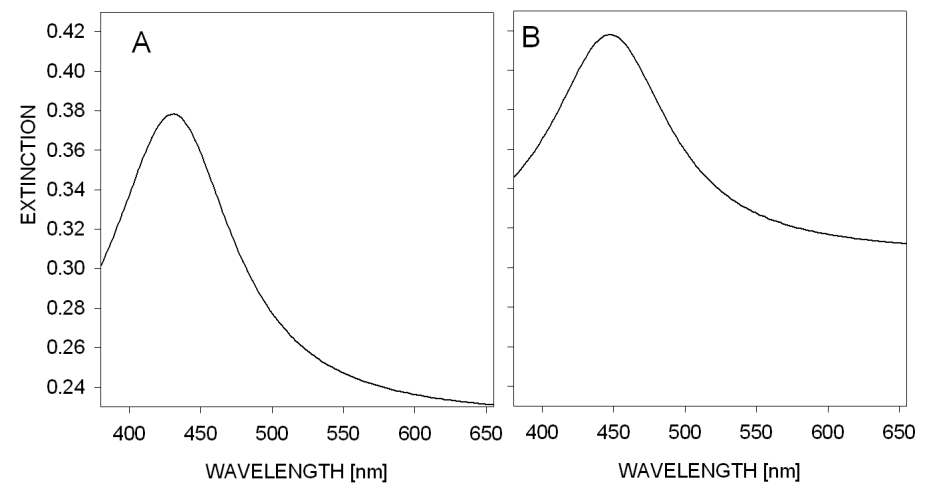

Fig. 4. Extinction spectra of silver island films obtained from colloid I (A) and colloid II (B).

tinction of this island film exhibits maximum at $450 \mathrm{~nm}$ i.e. at almost the same wavelength as colloid II. One should notice that at wavelengths larger than $500 \mathrm{~nm}$ extinction of this island film (obtained from colloid II) decreases slower than extinction observed for colloid II. It may prove that the aggregation of nanoparticles occurs during their deposition on glass. However, maximum of extinction of island film obtained from colloid II occurs almost at the same wavelength as for colloid II, then one can conclude that the aggregation of nanoparticles is not dominant.

Figure 5A presents fluorescence spectra of FITC diluted in (1) phosphate buffered saline (PBS) at pH 7.4, (2) 20-fold diluted colloid I with PBS and (3) 20-fold diluted colloid II with PBS. A slight increase in intensity of FITC fluorescence was observed when FITC was diluted in colloids and this increase depends on kind of colloid. Colloid II (nanoparticles radius $\approx 45 \mathrm{~nm}$ ) produces a slight larger than colloid I (nanoparticles radius $\approx 35 \mathrm{~nm}$ ) enhancement of fluorescence. Enhancement of fluorescence occurs when fluorescence molecules are placed near metallic nanoparticles but at distance beyond the range of quenching. Fluorescence molecules in the colloid can be placed in three regions: (I) very close to metallic nanoparticles, (II) at distance from nanoparticles preferable for MEF, and (III) at distance larger than preferable for MEF. Fluorescence of molecules situated in region I will be quenched, while the fluorescence of molecule from region II should be enhanced. Molecules placed in region III are neutral on the presence of metal nanoparticles and fluorescence should be the same as in water.

In the case of FITC molecules introduced into colloid, only their small part is placed in region II, and only the fluorescence of these molecules is enhanced, while the fluorescence of molecules situated in region I is quenched. The observed increase in fluorescence, as is shown in Fig. 5A, proves that in region II there must occur significant enhancement of fluorescence that exceeds the loss in intensity caused by quenching in region I. Results presented in Fig. $5 \mathrm{~A}$ seem to be little surprising. Our previous studies [27] proved the decrease in fluorescence of 


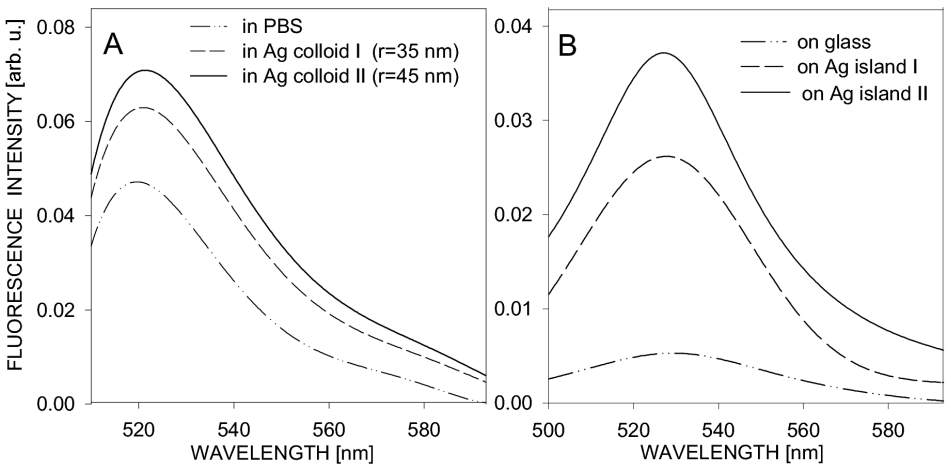

Fig. 5. (A) Fluorescence emission spectra of $0.1 \mu \mathrm{M}$ FITC in PBS at pH 7.4 and in 20-fold diluted by PBS colloid I and colloid II (both at pH 7.4). (B) Fluorescence emission spectra of FITC-HSA (9:1) layer deposited on glass slide and on silver island films obtained from colloid I and colloid II.

ochratoxin and camptothecin in the silver colloid. The observed discrepancies are most probably caused by the differences in fluorescence properties of FITC and previously studied compounds. FITC emits green light (maximum of emission at about $525 \mathrm{~nm}$ ), while camptothecin and ochratoxin violet-blue (maximum of emission at about $440 \mathrm{~nm}$ for both). Then the fluorescence band of FITC is beyond the colloid absorption band, while fluorescence of camptothecin and ochratoxin occurs exactly at the same spectral range as absorption of the colloid. Therefore, the fluorescence light of camptothecin and ochratoxin is absorbed by the colloidal nanoparticles while FITC fluorescence is not.

It is well known that fluorescence of FITC is self-quenched if FITC molecules are bound to proteins, e.g. HSA. Quenching increases as the labelling ratio FITC/HSA increases. The FITC-HSA mixture with labelling ratio 9:1 was chosen for studies. Figure 5B shows fluorescence spectra of FITC-HSA layer deposited on glass (dash-dot-dot line), on the silver island film obtained by glass dipping in colloid I (dash line) and on silver island film obtained by glass dipping in colloid II (solid line). Spectra of FITC diluted in water or in the colloid (Fig. 5A) and spectra of FITC-HSA layer (Fig. 5B) differ. Spectrum of FITC-HSA layer is shifted about $10 \mathrm{~nm}$ towards longer wavelengths. The observed changes in fluorescence spectra of FITC are caused by changes in the environment of FITC. Fluorescence intensity of FITC-HSA layer deposited on glass is very weak strong self-quenching occurs in FITC-HSA system. However, when FITC-HSA layer is deposited on silver island film, for FITC molecules, which are in distance of one HSA monolayer from silver nanoparticles, the conditions for fluorescence enhancement are fulfilled. Figure 5B shows that in the case of FITC-HSA layer deposited on silver island film obtained from colloid I (nanoparticles average radius $\approx 35 \mathrm{~nm}$ ), about 5-fold increase in fluorescence intensity was observed, meanwhile the same FITC-HSA layer deposited on silver island film obtained from colloid II 
(nanoparticles average radius $\approx 45 \mathrm{~nm}$ ) exhibited 7 -fold increase in the fluorescence intensity. There are several causes of stronger enhancement of fluorescence by silver island film obtained from colloid II. The most important are: (1) the cross section for scattering at wavelength of fluorescence, as is shown in Fig. 3B, is for film formed from colloid II a lot larger than cross-section for absorption, (2) frequency of FITC excitation $\left(\lambda_{\mathrm{exc}}=480 \mathrm{~nm}\right.$ ) is very close to the frequency at which $45 \mathrm{~nm}$ radius nanoparticles exhibit maximum of scattering (in the case of $35 \mathrm{~nm}$ radius nanoparticles this condition is not fulfilled - nanoparticles forming the colloid II exhibit maximum of scattering at $470 \mathrm{~nm}$, while nanoparticles forming Colloid I exhibit maximum of scattering at $440 \mathrm{~nm}$ ).

\section{Conclusions}

Fluorescence enhancement properties of the silver nanoparticles in colloids and silver nanoparticles forming island films on glass have been studied. Fluorescence of FITC in these two systems has been analyzed. Enhancement occurs, if fluorescence molecules are placed in the region preferable for MEF effect. Molecules adsorbed on silver nanoparticles and free molecules exist in the colloid. Fluorescence of these first ones is quenched, while only a part of free molecules is placed at the distance from silver nanoparticles enough good for MEF effect and therefore fluorescence of FITC dissolved in the silver colloids is only slightly higher than fluorescence of FITC diluted in water. In the case of FITC-HSA monolayer deposited on glass, number of fluorescence molecules in the field of excitation light is not large and additionally process of self-quenching occurs and therefore intensity of observed fluorescence is very weak. When the same FITC-HSA monolayer is deposited on the silver island film, the 5-7-fold increase in fluorescence intensity is observed. In the case of such FITC-HSA monolayer at least part of the fluorescence molecules is placed at the distance from metal preferable for MEF. For these molecules fluorescence enhancement occurs and this enhancement process exceeds the quenching and self-quenching of FITC fluorescence. The observed phenomenon of fluorescence enhancement is very promising. It may become very useful tool in the studies of low fluorescent compound, including biomolecules and biological systems, and may find application in biological and biomedical studies.

\section{References}

[1] M. Fleischman, P.J. Hendra, J. McQuillan, Chem. Phys. Lett. 26, 163 (1974).

[2] R. Aroca, Surface-Enhanced Vibrational Spectroscopy, Wiley, Chichester 2006.

[3] S. Kruszewski, Cryst. Res. Technol. 41, 562 (2006).

[4] H. Malak, in: The Teaching of the Physics in Technical Universities (Proc. Conf.), Ed. A. Gadomski, Academy of Technology and Agriculture, Bydgoszcz 2004, p. 15.

[5] D. Frąckowiak, A. Balter, J. Karolczak, P. Siejak, Acta Phys. Pol. A 110, 451 (2006). 
[6] J.R. Lakowicz, Analyt. Biochem. 298, 1 (2001).

[7] K. Aslan, I. Gryczynski, J. Malicka, E. Matveeva, J.R. Lakowicz, C.D. Geddes, Current Opinion in Biotechnology 16, 55 (2005).

[8] J.R. Lakowicz, J. Malicka, S. D’Auria, I. Gryczynski, Analyt. Biochem. 320, 13 (2003).

[9] S.L. Robia, N.C. Flohr, D.D. Thomas, Biochemistry 44, 4302 (2005).

[10] C.D. Geddes, J.R. Lakowicz, J. Fluorescence 12, 121 (2002).

[11] A. Derkachova, K. Kolwas, Eur. J. Phys. ST 144, 93 (2007).

[12] H. Ditlbacher, N. Felidj, J.R. Krenn, B. Lamprecht, A. Leitner, F.R. Aussenegg, Appl. Phys. B 73, 373 (2001).

[13] O. Stranik, H.M. McEvoy, C. McDonagh, B.D. MacCraith, Sensor Actuators B 107, 148 (2005).

[14] D. Cheng, Qing-Hua Xu, Chem. Commun. Iss. 3, 248 (2007), DOI: 10.1039/b612401a.

[15] E. Dulkeith, A.C. Morteani, T. Niederechholz, T.A. Klar, J. Feldmann, Phys. Rev. Lett. 89, 203002 (2002).

[16] O.G. Tovmachenko, Ch. Graf, D.J van den Heuvel, A. van Blaaderen, H.C. Gerritsen, Adv. Matter 18, 91 (2006).

[17] K. Aslan, Z. Leonenko, J.R. Lakowicz, C.D. Geddes, J. Fluorescence 15, 643 (2005).

[18] Y. Zhang, K. Aslan, S.N. Malyn, C.G. Geddes, Chem. Phys. Lett. 427, 432 (2006).

[19] J.R. Lakowicz, Analyt. Biochem. 337, 171 (2005).

[20] C.D. Geddes, I. Gryczynski, J. Malicka, Z. Gryczynski, J.R. Lakowicz, J. Fluorescence 14, 119 (2004).

[21] J. Lukomska, J. Malicka, I. Gryczynski, Z. Leonenko, J.R. Lakowicz, Biopolymers 77, 31 (2005).

[22] J. Aubard, E. Bagnasco, J. Pantigny, M.F. Ruasse, G. Levi, J. Phys. Chem. 99, 7075 (1995).

[23] J. Lukomska, J. Malicka, I. Gryczynski, J.R. Lakowicz, J. Fluorescence 14, 417 (2004).

[24] C.H. Munro, W.E. Smith, M. Garner, J. Clarkson, P.C. White, Langmuir 11, 3712 (1995).

[25] M.V. Cañamares, J.V. Garcia-Ramos, J.D. Gómez-Varga, C. Domingo, S. Sanchez-Cortes, Langmuir 21, 8546 (2005).

[26] J. Yguerabide, E.E. Yguerabide, Analyt. Biochem. 262, 137 (1998).

[27] S. Kruszewski, H. Malak, B. Ziomkowska, M. Cyrankiewicz, Proc. SPIE 6158, 61580 (2006) 